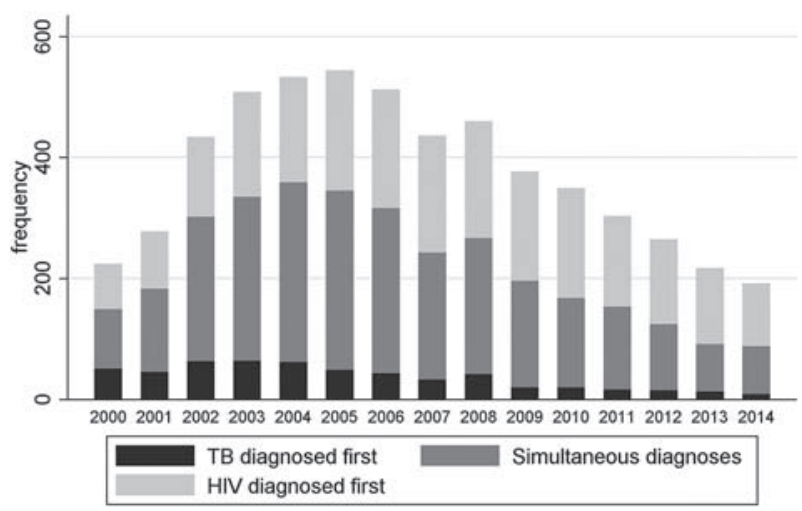

Abstract S28 Figure 1 First diagnosis for TB-HIV co-infected patients, 2000-2014.

Results 106,829 TB cases aged $\geq 15$ years were notified to PHE between 2000 and 2014, of which 5792 (5.4\%) were co-infected with HIV. The absolute number of HIV-TB diagnoses rose between 2000-2004, and then decreased from 543 $(8.0 \%)$ in 2004 to 205 (3.2\%) in 2014. Overall, 2,787/5,792 (49\%) were diagnosed with TB and HIV simultaneously, whilst 549 (9\%) were diagnosed with TB before and 2456 (42\%) after HIV diagnosis. The relationship between TB and HIV diagnosis changed over time (figure 1). The absolute number of $\mathrm{TB}$ cases in people with known HIV rose from $75 / 224(33 \%)$ in 2000 to $210 / 454(46 \%)$ in 2007 , then fell to $117 / 205(57 \%)$ by 2014 , but increased as a proportion of all HIV-TB cases from 2000 to 2014. There were corresponding declines in the proportion diagnosed simultaneously with TB and HIV from 298/543 (55\%) in 2004 to $79 / 205$ (39\%) in 2014 and those first diagnosed with TB (51/224 (23\%) in 2000 to $9 / 205(4 \%)$ in 2014).

Conclusions Within an overall decline in HIV-TB co-infection there has been a change in the pattern of co-infection. A greater proportion of cases now occur in people with known HIV infection, and fewer HIV infections are diagnosed after TB diagnosis. This may be explained by more HIV testing, including in $\mathrm{TB}$ clinics, resulting in earlier HIV diagnosis. However, as the number of people with HIV in the UK increases, sustained success requires better management of latent $\mathrm{TB}$ infection to prevent the occurrence of $\mathrm{TB}$ disease in people diagnosed with HIV.

\section{S29 A RANDOMISED CONTROLLED TRIAL COMPARING SMARTPHONE ENABLED REMOTE VIDEO OBSERVATION WITH DIRECT OBSERVATION OF TREATMENT FOR TUBERCULOSIS}

${ }^{1} \mathrm{~A}$ Story, ${ }^{1} \mathrm{R}$ Aldridge, ${ }^{1} \mathrm{C}$ Smith, ${ }^{2} \mathrm{E}$ Garber, ${ }^{2} \mathrm{~J}$ Hall, ${ }^{2} \mathrm{G}$ Fernandez, ${ }^{2} \mathrm{~L}$ Possas, ${ }^{2} \mathrm{~S}$ Hemming, ${ }^{2} \mathrm{M}$ Coxsedge, ${ }^{1} \mathrm{~F}$ Wurie, ${ }^{1} \mathrm{~S}$ Luchenski, I Abubakar, ${ }^{1} \mathrm{~T}$ McHugh, ${ }^{3} \mathrm{P}$ White, ${ }^{1} \mathrm{JM}$ Watson, ${ }^{2} \mathrm{M}$ Lipman, ${ }^{4} \mathrm{R}$ Garfein, ${ }^{1} \mathrm{~A}$ Hayward. 'University College London, London, UK; ${ }^{2}$ Royal Free London NHS Foundation Trust, London, UK; ${ }^{3}$ Imperial College, London, UK; ${ }^{4} U C S D$, San Diego, US

\subsection{6/thoraxjnl-2017-210983.35}

Background Directly observed treatment (DOT) has been the standard of care for tuberculosis since the early 1990s. In England DOT is targeted at those considered to be at high risk of poor adherence and clinically complex patients. We report the first randomised controlled trial of smartphone- enabled video observation of treatment (VOT) for active tuberculosis compared to DOT.

Methods Tuberculosis patients eligible for selective DOT in England were randomised to an offer of asynchronous VOT (daily remote observation using a smartphone app) or DOT (3 or 5 times weekly observation in community or clinic settings).

Results $58 \%$ of 226 randomised patients had a history of homelessness, drug use, imprisonment, alcohol or mental health problems. Of the 112 patients randomised to an offer of VOT, $70 \%$ had over $80 \%$ of scheduled observations completed over two months (the primary outcome measure) compared to $31 \%$ of 114 patients randomised to an offer of DOT $(p<0.001)$. The effect was, in part, due to $51 \%$ of those randomised to DOT having less than one week of observation (compared to $10 \%$ of those randomised to VOT), and so not starting treatment with their allocated regimen. VOT patients sustained high observation levels throughout treatment, whereas this declined rapidly in DOT patients. We estimate that observation of a six month course of treatment with daily VOT cost $£ 1645$ per patient compared to $£ 5700$ for five times per week DOT.

Conclusions VOT is a more effective and cheaper approach to observation of tuberculosis treatment than clinic or community based DOT.

\section{S30 IS THE NEW NATIONAL LTBI SCREENING PROGRAM REACHING THE TARGET POPULATION? A POPULATION- BASED COHORT STUDY}

${ }^{1} \mathrm{LC}$ Berrocal-Almanza, ${ }^{2} \mathrm{AM}$ OConnell, ${ }^{2} \mathrm{MC}$ Muzyamba, ${ }^{2} \mathrm{~A}$ Mirza, ${ }^{2} \mathrm{M}$ Lalor, ${ }^{1} \mathrm{~A}$ Lalvani, ${ }^{2} \mathrm{D}$ Zenner. ${ }^{1}$ Imperial College London, National Institute for Health Research Health Protection Research Unit in Respiratory Infections, London, UK; ${ }^{2}$ Public Health England (PHE), Centre for Infectious Disease Surveillance and Control, London, UK

\subsection{6/thoraxjnl-2017-210983.36}

In England (73\%) of $\mathrm{TB}$ cases occur among foreign-born individuals due to reactivation of Latent $\mathrm{TB}$ infection (LTBI). ${ }^{1}$ The cornerstone of the global strategy to eliminate $\mathrm{TB}$ in low-incidence countries is to target preventative therapy at high-risk groups of developing active disease. England is the first country implementing a $£ 10 \mathrm{~m} /$ annum program to systematically provide LTBI screening and treatment for new entrant migrants. $^{2}$ The main challenge is how to identify migrants eligible for screening among the reservoir of migrants already registered and those unregistered with primary-care. We aimed to establish the most feasible and effective method to localise migrants eligible for LTBI screening and to estimate population size, TB incidence after arrival and LTBI service utilisation of the target population. We performed a retrospective population based cohort study of VISA-applicants aged 16 to 35 years screened for active TB before entry to UK in 72 high-burden countries between 2011 and 2014. We used data record linkage of the pre-entry screening, the NHS Personal Demographic Service (PDS), the Enhanced TB Surveillance system and LTBI service utilisation databases. 193979 migrants eligible for LTBI screening entered the UK between 2011 and 2014. A low proportion $78395(40.4 \%)$ registered in primary-care in the year of arrival, of whom 30838 (39.3\%) registered in high TB incidence areas of London and Midlands. We estimated the number of delayed registrations of the same age group in these areas by record linkage; 
160780 immigrants from the 72 high-burden countries registered between 2011 and 2014, of whom 129942 (80.8\%) arrived before 2011. These delayed registrants thus miss the window of opportunity for LTBI screening because the program targets arrivals within last five years. We estimated and compared TB incidence and LTBI service utilisation in the cohort of early vs delayed registrants. The program may be compromised by low and delayed primary-care registration, could be enhanced by promoting GP registration and community-based screening to reach unregistered migrants.

\section{REFERENCES}

1. Public Health England. Tuberculosis in England 2016.

2. Public Health England. Collaborative Tuberculosis Strategy for England 20152020.

\section{S31 PROGNOSTIC VALUE OF INTERFERON GAMMA RELEASE ASSAYS AND TUBERCULIN SKIN TEST IN PREDICTING THE DEVELOPMENT OF ACTIVE TUBERCULOSIS: THE UK PREDICT TB COHORT STUDY}

\begin{abstract}
${ }^{1}$ I Abubakar, ${ }^{2} \mathrm{~F}$ Drobniewski, ${ }^{3} \mathrm{~J}$ Southern, ${ }^{4} \mathrm{AJ}$ Sitch, ${ }^{1} \mathrm{C}$ Jackson, ${ }^{1} \mathrm{M}$ Lipman, ${ }^{1} \mathrm{JJ}$ Deeks, ${ }^{5} \mathrm{C}$ Griffiths, ${ }^{6} \mathrm{G}$ Bothamley, ${ }^{7} \mathrm{~W}$ Lynn, ${ }^{8} \mathrm{H}$ Burgess, ${ }^{8} \mathrm{~B}$ Mann, ${ }^{1} \mathrm{~A}$ Imran, ${ }^{2} \mathrm{~S}$ Sridhar, ${ }^{3} \mathrm{CY}$ Tsou, ${ }^{3} \mathrm{~V}$ Nikolayevskyy, ${ }^{2} \mathrm{M}$ Rees-Roberts, ${ }^{2} \mathrm{H}$ Whitworth, ${ }^{2} \mathrm{O}$ Min Kon, ${ }^{9} \mathrm{P}$ Haldar, ${ }^{5} \mathrm{H}$ Kunst, ${ }^{3} \mathrm{~S}$ Anderson, ${ }^{1} \mathrm{~A}$ Hayward, ${ }^{3} \mathrm{JM}$ Watson, ${ }^{10} \mathrm{H}$ Milburn, ${ }^{2} \mathrm{~A}$ Lalvani. ${ }^{1}$ University College London, London, UK; ${ }^{2}$ Imperial College, London, UK; ${ }^{3}$ Public Health England, London, UK; ${ }^{4}$ University of Birmingham, Birmingham, UK; ${ }^{5}$ Queen Mary University of London, London, UK; ${ }^{6}$ Homerton University Hospital, London, UK; ${ }^{7}$ Ealing Hospital, London, UK; ${ }^{8}$ West Middlesex Hospital, London, UK; ${ }^{9}$ University of Leicester, Leicester, UK; ${ }^{10}$ Guys and St Thomas' Hospital, London, UK
\end{abstract}

\subsection{6/thoraxjnl-2017-210983.37}

Background Tackling tuberculosis (TB) requires testing and treatment of high-risk groups for latent tuberculosis infection. We estimated the predictive values of the tuberculin skin test (TST) and interferon gamma release assays (IGRAs) for development of active $\mathrm{TB}$ in migrants and contacts of active $\mathrm{TB}$ patients in the UK.

Methods Participants were prospectively recruited in clinics and the community and followed for a median of 2.9 years. We administered IGRAs (Quantiferon Gold In-Tube [QFT-GIT] and T-SPOT.TB) and TST (with 3 thresholds: $5 \mathrm{~mm}\left(\mathrm{TST}^{5}\right.$ ), $10 \mathrm{~mm}\left(\mathrm{TST}^{10}\right)$ and $\mathrm{TST}^{15}$ (5 mm in BCG-naïve, $15 \mathrm{~mm}$ in vaccinated). Potential incident TB cases were identified by telephone interview and national $\mathrm{TB}$ databases and confirmed by medical note review.

Results Ninety-seven (1.0\%) of 9610 participants developed active TB (77 of 6386 who had Results for T-SPOT.TB, QFTGIT and TST). All tests had very low incidence in test negatives (1.2-1.6 per 1000 per year). Incidence rates in test positives were highest for TSpot.TB (13.2 95\% CI: (9.9-17.4)), $\operatorname{TST}^{15}(11.1(8.3,14.6))$ and QFT.GIT $(10.1(7.4,13.4))$; positive test Results for these tests were significantly more predictive of progression than $\mathrm{TST}^{10}$ and $\mathrm{TST}^{5}$, TSpot.TB was also higher than QFT.GIT. TST $^{5}$ predicted more at high risk $(55 \%)$ than $\mathrm{TST}^{10}$ (45\%), TSpot.TB (33\%), $\mathrm{TST}^{15}$ (38\%) and QFT.GIT (31\%).

Conclusions IGRA-based or TST $^{15}$ strategies are most suited for population screening in low-risk populations. Although $\mathrm{TST}^{5}$ and $\mathrm{TST}^{10}$ detect more TB cases this is at the cost of more individuals being classified at high risk with lower positive predictive values.

\section{A troublesome cough: from diagnosis to treatment}

\section{$\$ 32$ COUGH SUPPRESSION TEST: A NOVEL OBJECTIVE TEST FOR CHRONIC COUGH}

${ }^{1} \mathrm{PSP}$ Cho, ${ }^{2} \mathrm{H}$ Fletcher, ${ }^{2} \mathrm{RD}$ Turner, ${ }^{1,2} \mathrm{SS}$ Birring. 'Division of Asthma, Allergy and Lung Biology, King's College London, London, UK; ${ }^{2}$ King's College Hospital NHS Foundation Trust, London, UK

\subsection{6/thoraxjn|-2017-210983.38}

Introduction A recent functional MRI study has shown that patients with chronic refractory cough (CRC) have reduced activity in the areas of the brain associated with cough suppression. Cough challenge tests focus only on provoking cough and have limited clinical application due to the wide overlap between healthy subjects and patients with cough. We investigated whether patients with CRC could suppress cough in a cough challenge test.

Methods We recruited 13 chronic refractory cough patients and 11 healthy controls. Participants underwent an incremental capsaicin challenge test $\left(0.49\right.$ to 1000 micromol. $\left.\mathrm{L}^{-1}\right)$ and were instructed "please do not cough during the test". The concentrations of capsaicin during the cough suppression (CS) protocol required to elicit 1 or more cough (CS1), 2 or more coughs (CS2), and 5 or more coughs (CS5) were documented. Patients with CRC also completed cough-severity and urge-tocough visual analogue scales (VAS; $0-100 \mathrm{~mm}$ ), and quality of life, Leicester Cough Questionnaire (LCQ; range 3-21).

Results Patients with CRC and controls had a mean (SD) age 57 (8) and 51 (7) years and $11(85 \%)$ and 7 (64\%) were female, respectively. CRC patients self-reported symptom and health status were; mean (SD) cough severity VAS 58 (31), urge-to-cough VAS 63 (30), and LCQ score 12.1 (4.4). Patients with CRC were less able to suppress cough compared to healthy controls; geometric mean (SD) CS1: 2.30 (3.56) vs 62.46 (5.62), CS2: 2.55 (3.71) vs 70.86 (5.91) and CS5: 3.37 (4.84) vs $321.70 \quad$ (3.23) micromol. $\mathrm{L}^{-1}$ respectively, all $\mathrm{p}<0.0001$. The mean difference $(95 \% \mathrm{CI})$ in CS5 between CRC and controls was $6.6(4.9,8.3)$ doubling doses. CS5 was better than CS1 and CS2 at discriminating CRC patients from controls (figure 1). There was no significant association between CS5 and cough severity VAS (correlation coefficient,

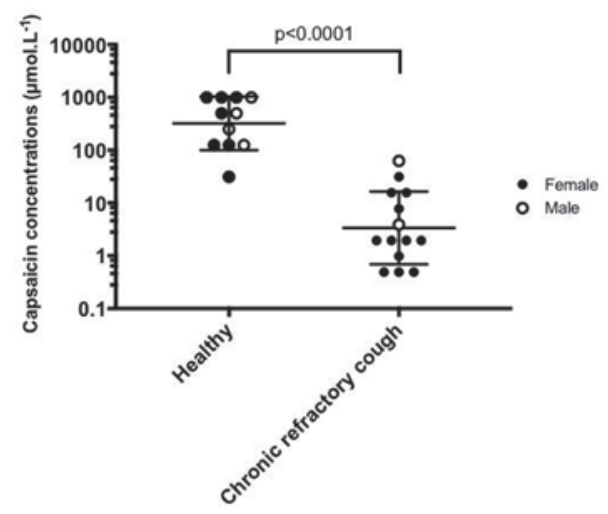

Abstract S32 Figure 1 Cough suppression test. Capsaicin concentration (geometric mean, SD) that provoked 5 or more coughs (CS5) during voluntary suppression of cough. 